\title{
Smart Antennas in Software Radio Base Stations
}

\author{
Ana Pérez-Neira, Xavier Mestre, and Javier Rodríguez Fonollosa, Universitat Politècnica de Catalunya
}

\begin{abstract}
The application of adaptive antenna techniques to fixed-architecture base stations has been shown to offer wide-ranging benefits, including interference rejection capabilities or increased coverage and spectral efficiency. Unfortunately, the actual implementation of these techniques to mobile communication scenarios has traditionally been set back by two fundamental reasons. On one hand, the lack of flexibility of current transceiver architectures does not allow for the introduction of advanced add-on functionalities. On the other hand, the often oversimplified models for the spatiotemporal characteristics of the radio communications channel generally give rise to performance predictions that are, in practice, too optimistic. The advent of software radio architectures represents a big step toward the introduction of advanced receive/transmit capabilities. Thanks to their inherent flexibility and robustness, software radio architectures are the appropriate enabling technology for the implementation of array processing techniques. Moreover, given the exponential progression of communication standards in coexistence and their constant evolution, software reconfigurability will probably soon become the only costefficient alternative for the transceiver upgrade. This article analyzes the requirements for the introduction of software radio techniques and array processing architectures in multistandard scenarios. It basically summarizes the conclusions and results obtained within the ACTS project SUNBEAM, ${ }^{1}$ proposing algorithms and analyzing the feasibility of implementation of innovative and softwarereconfigurable array processing architectures in multistandard settings.
\end{abstract}

\section{INTRODUCTION}

The increasingly fragmented nature of the international mobile communications market (illustrated by the growing number of standards) presents an opportunity for manufacturers to develop base station equipment able to support a wide range of air interfaces.
Progress in digital signal processing component technology is offering the possibility of highly flexible radios, where most of the signal processing is performed in software and reconfigurable digital hardware. It is therefore in this field that the software radio concept is emerging as a potential pragmatic solution. In fact, the term software radio stands for radio functionalities defined by software, meaning the possibility to software-define the typical functionality of a radio interface, usually implemented in transmitter and receiver equipment by dedicated hardware.

Smart antennas emerge as the enabling technology that exploits the available resources of the physical layer, which is the basis of the whole mobile communication concept. Future base stations, which aim to support multiple air interface standards, will require beamforming architectures. In order to exploit the software radio concept, the array processing subsystem should not become an impediment to achieving fully flexible base station architectures. Therefore, there is a need to develop beamforming architectures and algorithms, which will allow the benefits of adaptive antennas to be fully exploited in future multistandard base stations. Software radio constitutes the key technology for the potential implementation of such flexible base stations, but the potential implications of its application to array processing configurations need to be further analyzed. This was the main objective of the Smart Universal Beamforming (SUNBEAM) project.

SUNBEAM was carried out under the umbrella of the Advanced Communications Technologies and Services (ACTS) Program and builds on the work of the ACTS Technology in Smart Antennas for Universal Advanced Infrastructure (TSUNAMI) (II) project. TSUNAMI(II) ran from September 1995 to June 1998 and was basically concerned with the application of adaptive antennas to third-generation systems such as Universal Mobile Telecommunications System (UMTS). In accordance with the spirit of the ACTS Program, the TSUNAMI (II) project placed a strong emphasis on building a demonstrator and using it in a field trial. The equipment was deployed within 
the premises of an operational mobile network of a national host in the United Kingdom. The SUNBEAM project further developed TSUNAMI(II) by identifying algorithms and architectures which enable adaptive antenna techniques to be exploited in flexible multistandard UMTS/Global System for Mobile Communications (GSM) base stations.

The SUNBEAM consortium was formed by 11 organizations, including six industrial partners (ERA Technology - project coordinator, Analog Devices Ltd., Robert Bosch GmbH, Motorola ECID, Wireless Systems International, and Thomson CSF Communications), one operator (France Telecom CNET), and four universities (Ecole Nationale Superieure des Telecommunications, Aalborg University, University of Bristol, and Universitat Politècnica de Catalunya). Most of the partners were already participants in the TSUNAMI (II) project, so SUNBEAM was originally conceived to build on the work of its predecessor, taking advantage of the knowledge already gained in previous collaborations.

Figure 1 represents the work package breakdown of the SUNBEAM project. After a study of the market requirements to set the proper focus of the project, five different working lines were established: radio frequency (RF) architectures, 2D radio channel characterization, algorithm development, system simulations, and implementation of a software radio prototype.

One of the main objectives of the project was to demonstrate the implementation of array processing functions in pseudo real time on a software radio platform. The array processing algorithms implemented were first developed and tested in link- and system-level simulations. In order to obtain a realistic assessment of the performance-complexity trade-off of the different algorithms, first at the link and system simulator and then at the software radio platform, it was extremely important that the channel impulse responses simulated be as realistic as possible. For this reason, a set of measurement campaigns was carried out in order to study and propose suitable models for the spatiotemporal characteristics of the radio channel. In parallel with this activity, the design of the

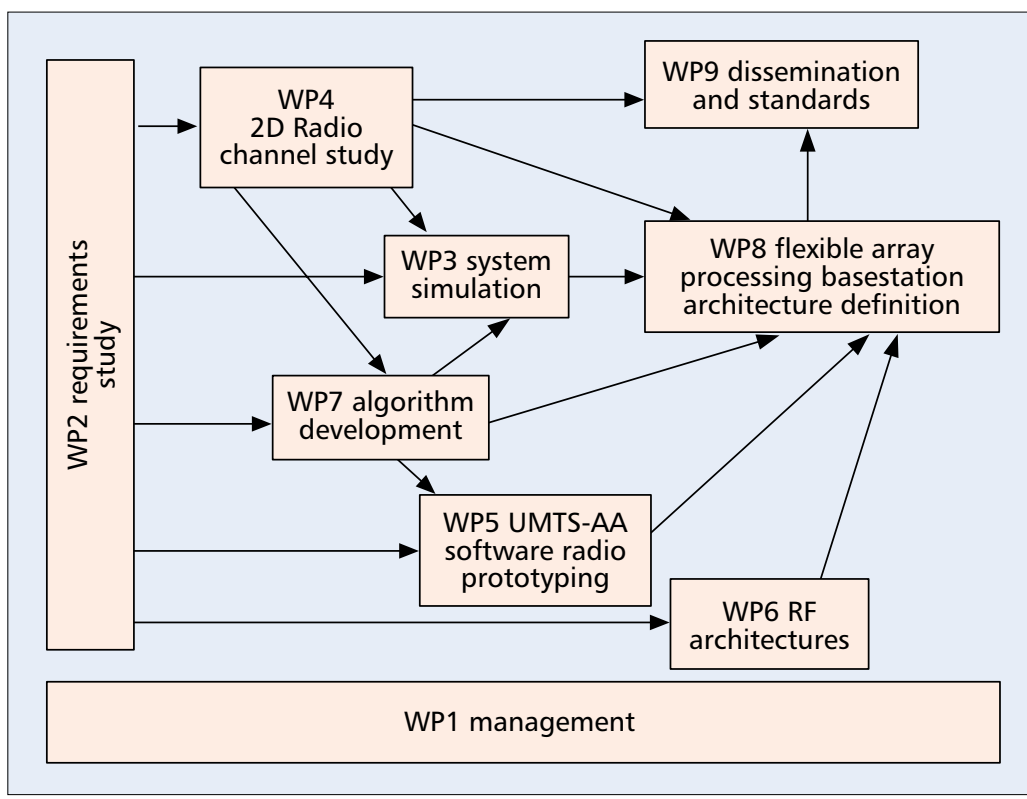

Figure 1. The work package structure of the SUNBEAM project.

RF transceiver and the structural implications of this design on the software radio platform were also analyzed.

The complementary nature of the project activities enabled assessment from a global perspective of the viability of software radio architectures for wideband multistandard array processing base stations. As an example of the architectural analysis carried out in the SUNBEAM project, Fig. 2 outlines the global architecture of the wideband base station designed to process multicarrier inputs. The development of a software radio system implies, above all, the achievement of two main goals:

- To move, in transmission and reception, the boundary between the analog and digital worlds as much as possible toward radio frequency

- To replace application-specific integrated circuits (ASICs) with digital signal processors (DSPs) for baseband signal processing

Given these two goals, this article is mainly devoted to highlighting the architectural considerations of: the RF transceiver, the multicarrier

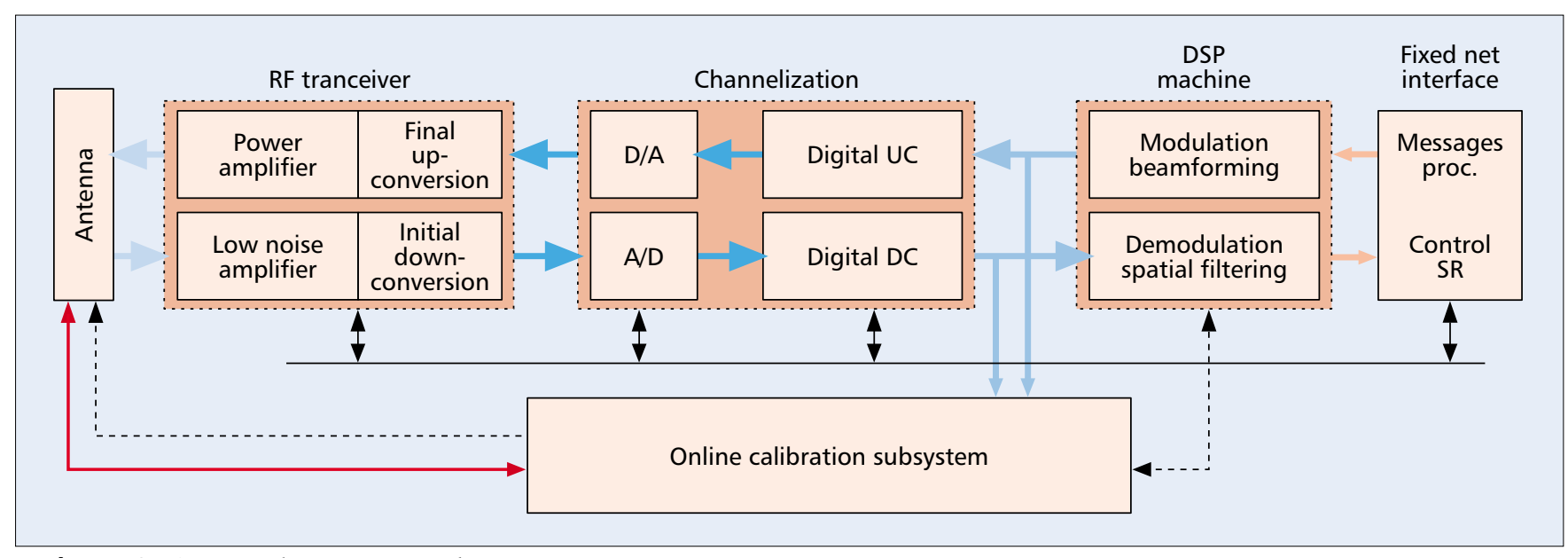

Figure 2. A generic base station architecture. 
channelization module, and the baseband transceiver. This latter item will mainly describe the design of a global reconfigurable array processing algorithm.

\section{THE RF TRANSCEIVER AND Channelization for Software Radio}

One fundamental aspect of the base station architecture, especially when adaptive antennas are considered, concerns the design of the physical antenna itself. Multistandard receive and transmit requirements significantly enlarge the RF bands used by the systems to be implemented. This fact motivated special interest in the design of antenna elements with a radiation pattern nearly frequency-independent. After initial experiments with stacked patch antennas, the final approach taken by Bosch engineers was an array of four planar notch antenna elements. The resulting prototype was able to operate over the bands of DCS1800 (1710-1880 MHz) and UMTS (1880-2170 MHz) with linear vertical polarization. The measured beam pattern was almost constant over the entire frequency band under consideration, offering a wide

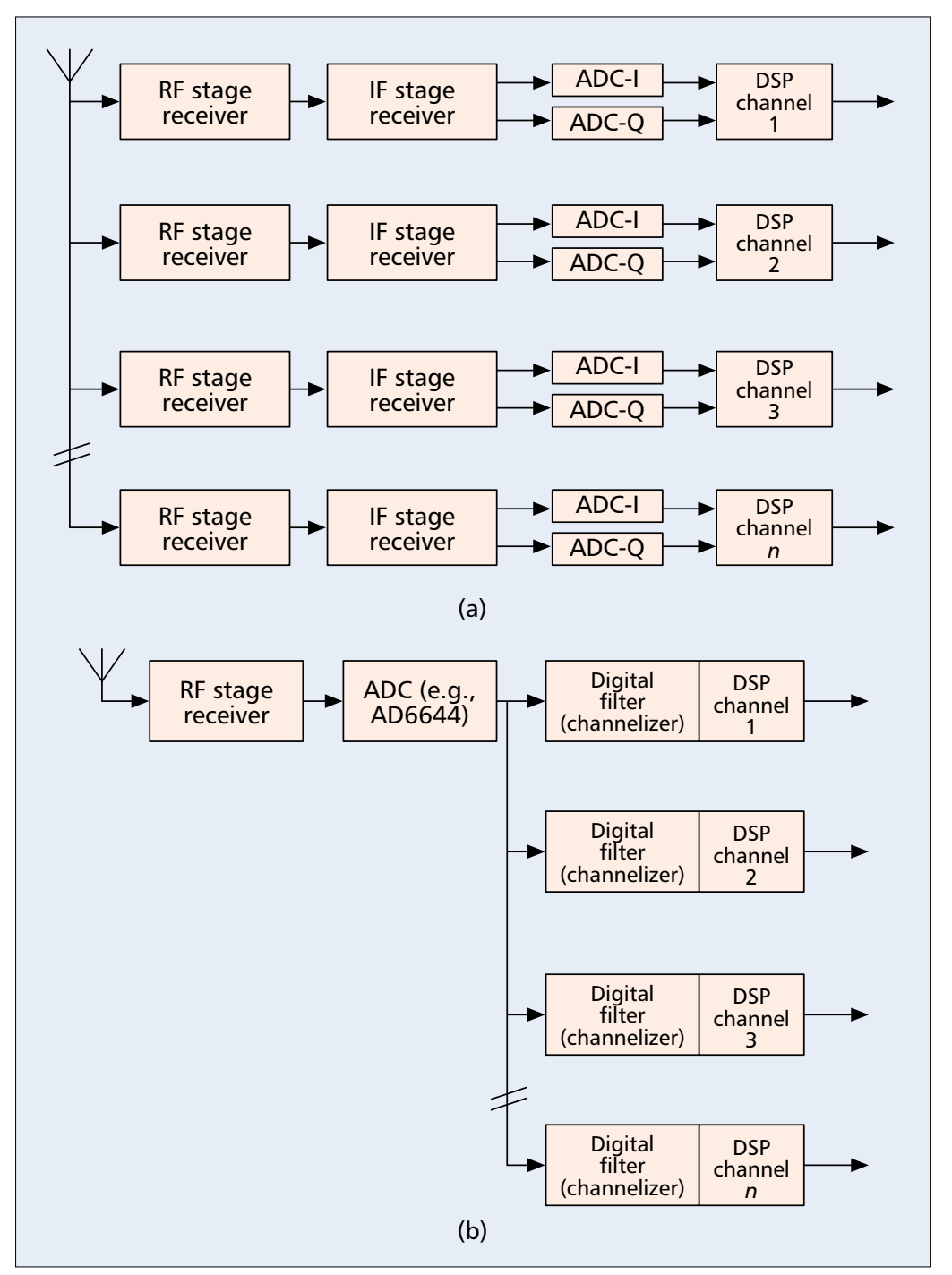

Figure 3. Multicarrier receiver architecture using a) traditional architecture; b) a wideband IF sampling $A D C$. beamwidth in the azimuth plane (about $144^{\circ}$ ) and a beam angle of $35^{\circ}$ in the elevation plane. Thanks to its high bandwidth, high azimuth beamwidth, and good frequency independence, the designed antenna was considered very appropriate for applications to multistandard configurations.

One of the main objectives of the SUNBEAM project was to analyze different front-end architectures and assess the feasibility of their implementation taking into account current specifications of wideband mixed-signal technology. Several architectural trends can be identified, the basic difference among them being the position of the data converters within the transceiver. For instance, let us consider the reception of several users not necessarily transmitting with the same modulation formats. Two different reception strategies can in principle be envisaged (Fig. 3). In a traditional multichannel design, such as that shown in Fig. 3a, the entire receiver is replicated for each channel, yielding an overly redundant design. Each added analog stage in a receiver adds expense due to yield, alignment, and unpredictability. In this sense, the sooner the desired signal is digitized, the better. Once digitized, the signal can be tuned, filtered, and conveniently processed. This is exactly the philosophy followed by the architecture in Fig. 3b: a single RF downconverter followed by a single IF sampling wideband analog-digital converter (ADC) has replaced the multiple front-ends, thereby saving time and cost in manufacture. The output of the ADC consisting of the digitized spectral data is then fed to a digital filter bank in charge of channelization (i.e., selection of the carrier and channel to be elaborated by digital filtering). Many passive discrete and analog components that formed the tuning and filtering functions have been eliminated in Fig. 3b. These components often required "tweaking" and special handling during manufacture. Digital components require no such adjustments, since tuner and filter characteristics are always exactly the same and do not vary with the fabrication process. Additionally, tuning and filtering characteristics can be changed through software.

The architectures shown in Fig. 3a and b correspond to two different data conversion strategies, respectively:

- A traditional baseband sampling technique, used by the well-known superheterodyne receiver

- An intermediate frequency (IF) sampling technique, where sampling is performed prior to baseband conversion

The same two strategies can be applied at the transmitter, depending on whether conversion to IF is made prior to or after the digital-analog conversion (DAC). The second alternative eliminates the need for a tuning local oscillator, and provides a highly modular and reconfigurable architecture, very suitable for software radios. That is, additional channels can easily be accommodated by the incorporation of digital tuner ICs, adding greatly to the reliability of the system because, unlike its analog counterpart, this digital technology requires no adjusting during the manufacturing process. 
However, the IF sampling approach imposes stronger constraints on all the processing stages prior to downconversion at the receiver and after upconversion at the transmitter. The price associated with the cutback in the number of channels (thanks to the IF sampling) is the widening of the remaining channel bandwidth. The complexity reduction comes at the expense of more stringent linearity requirements for the amplification components in the RF/IF section prior to the ADC and after the DAC, since all the conversion gain can only be given to the ana$\log$ wideband signal. Therefore, to handle broader signal bandwidth, new emerging technologies have been investigated in the wideband transceiver and channelization block: linearized multicarrier power amplifiers (up to $30 \mathrm{MHz}$ linearization bandwidth) and high-speed highdynamic-range $\mathrm{ADC} / \mathrm{DAC}$ ( $>60$ Msamples/s) in the channelization block.

The need for highly linear components at the $\mathrm{RF} / \mathrm{IF}$ stages is particularly crucial in the transmitter, since adjacent channel emissions are highly unwanted. This fact motivated the study of linearization techniques based on simple and power-efficient predistortion devices, implemented at RF, IF, or baseband level. These techniques prove extremely useful in that they will facilitate the use of power-efficient class $C$ amplifiers on signals modulated with nonconstant envelope, such as those proposed by thirdgeneration standards. Predistortion devices basically implement nonlinear transfer functions that are designed to compensate for the transmitter nonlinearities. They are capable of multioctave operation and provide high degrees of linearity improvement over a reasonable linearization bandwidth.

One of the most critical aspects determining the performance and feasibility of implementing software radios concerns the performance requirements of the data conversion devices of the transceiver. In software radio applications for second- and third-generation communication standards, ADCs must give sample rates well

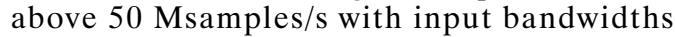
larger than $100 \mathrm{MHz}$, a high effective number of bits (defined as the number of bits of an ideal $\mathrm{ADC}$ to obtain the same performance as the real ADC), and low aperture jitters (defined as the uncertainty about the sampling instant). Note that these converters are normally used in bandpass sampling configurations, where the bandpass signal is sampled at a rate much lower than the carrier frequency so that the aliasing effect is used as a downconversion mechanism. Considering the high frequency and large bandwidth of the signals to process, only dedicated circuits could reach the required CPU power at reasonable costs. Therefore, new specific ASICs have been developed to support this new digital transceiver concept, taking advantage of the emerging technologies. This is the case of Analog Devices products, which cover consistently either needs for digital-to-analog or IF-to-baseband conversions. For instance, the final downconversion could in principle be provided by the AD6620. This is a digital decimating receiver or digital receive signal processor (DRSP) designed to digitally downconvert the signal to baseband.
Finally, as mentioned above, the wideband IF sampling ADC architecture of Fig. 3b eliminates the need for multiple front-ends, providing large savings. Thus, the incremental cost for each channel is only that of the digital filter, not of the entire radio front-end. Until quite recently, high-performance converters demanded a very large premium in cost, but as new converter architectures and silicon processes have developed, these price premiums have been reduced considerably. As shown above, the bulk of all hardware cost in a multichannel receiver is shared between channels. A recent study of cost models illustrated that on a per-channel basis, the cost per channel quickly falls below that of a traditional narrowband receiver. Furthermore, as new channels are added to this receiver architecture, the cost per channel falls as well. These are basically the reasons multichannel receivers can provide a noteworthy cost advantage.

To complete the description of the reconfigurable channelization stage, we comment that for a wideband transmit architecture, the backend has been replaced with multiple digital transmit signal processors (DTSPs) which perform pulse shaping, interpolation, digital filtering, and finally digital quadrature upconversion to IF before being applied to a single high-speed wideband DAC (such as the 14-bit AD9754). The basic limitation for DAC implementation in multitone applications is that DAC spurious performance must be excellent since there is no ability to clean up DAC spurs in-band due to its wideband application.

A fundamental issue in the development of a multistandard smart antenna base station is the design of a reconfigurable baseband DSP. The next section is devoted to the design of sufficiently flexible array processing algorithms.

\section{Channel Sounding and Flexible Algorithms for Baseband Processing}

Concerning the software implementation of array processing architectures, field trials using an enhanced version the TSUNAMI (II) standalone testbed were performed, and the results were used to propose a statistical characterization of the spatiotemporal radio channel. In addition, new array processing algorithms for third-generation mobile communication standards were designed, and their performance was evaluated using the realistic channel models obtained in the channel sounding activity.

If advanced multi-antenna systems are to be properly designed and assessed, a deep investigation of the space and time characteristics of the channel is mandatory. In that respect, while the time and frequency properties of the mobile radio channel are well documented in the literature, little attention has been paid to its spatial characterization. Note that antenna arrays make use of a spatial sampling of the received signal, and that this fact adds a new component to the two classical dimensions of the mobile radio channel (time/frequency). In general terms, the signal coming from a mobile station is seen by the base station as a distributed

\section{A fundamental \\ issue in the \\ development of a \\ multi-standard \\ smart-antenna \\ base-station is \\ the design of a \\ reconfigurable \\ baseband digital \\ signal processor.}


High gains in

system capacity

can be expected

when using

antenna array

configurations

operating in

environments rich

in scatters such

as indoor

settings.

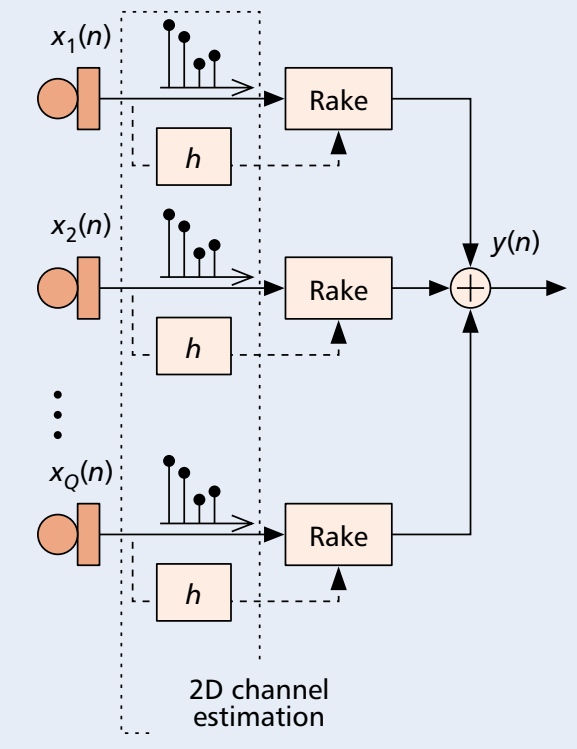

Matched Desired Impulse Response (MDIR) receiver

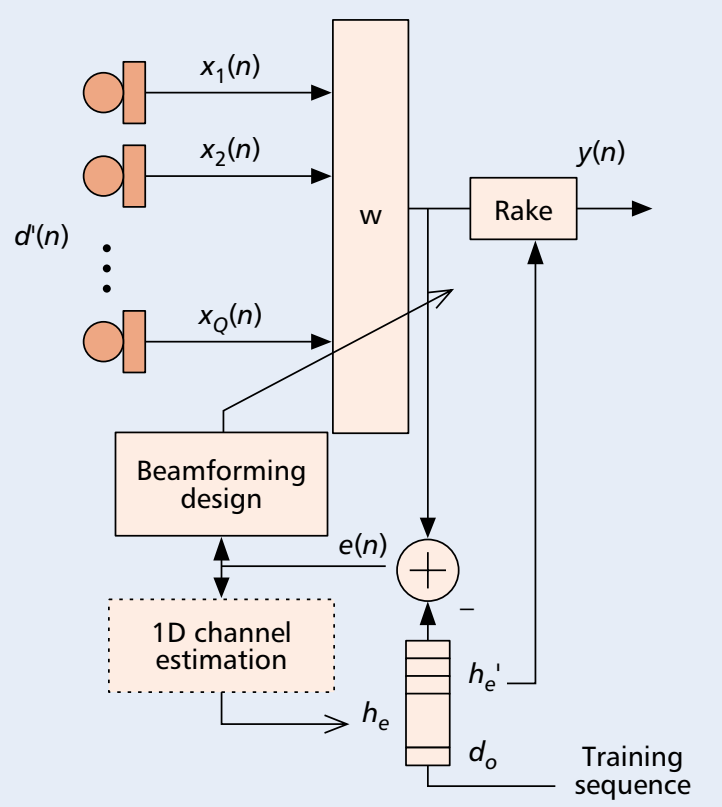

Figure 4. Uplink array processing algorithms under test.

source rather than a punctual one. In an attempt to characterize and find probabilistic models associated with the observed radio channel, an extensive campaign of channel measurements - already started during the TSUNAMI (II) project - was carried out by the Center of PersonKommunications of Aalborg University. According to the channel characterization, the spatial power distribution measured at the base station follows quite closely a Laplacian law centered at the actual physical direction of emission of the mobile station. Besides, the distribution of the directions of arrival (DOAs) associated with the different wavefronts impinging on the array was well approximated by a centered Gaussian distribution. This spatial model for the radio channel was later used to assess the analytical performance of different vector Rake receivers, to perform realistic system simulations of distinct array processing algorithms, and to synthesize the inputs to the software radio demonstrator.

The second part of the measurement campaign was focused on the characterization of mobile radio channels with multiple input and multiple output (MIMO). These measurements were carried out in order to give a realistic idea of the potential gain from using distinct antenna array configurations. From a theoretical perspective, it was found that the potential capacity improvement depended strongly on the correlation between antennas at both mobile and base station. Therefore, high gains in system capacity can be expected when using antenna array configurations operating in environments rich in scatters such as indoor settings. Further results on performance evaluation of MIMO systems including MIMO channel measurements have been obtained within the IST project Multi-Element Transmit and Receive Antennas (METRA) (http://www.ist-metra.org).
The array processing algorithms used in mobile communication environments are usually designed to operate in a particular air interface. Thus, the increasing number of communication standards motivates the investigation of array processing schemes that can be software-adapted to the different modulations and access techniques. The study conducted in SUNBEAM has been performed in two stages: first, the suitability of different algorithms for the systems under consideration is analyzed (UTRA-FDD, UTRA-TDD, and GSM), and a set of preferential array processing algorithms is selected for each mode of operation. This has been made via simulations making use of the realistic channel models derived in the project. Then the common functionalities among the different selected algorithms are identified, and a global reconfigurable array processing algorithm is finally proposed. This task has been carried out by Universitat Politècnica de Catalunya in cooperation with ERA Technology. The set of selected reconfigurable algorithms was implemented in a software radio prototype by Thomson CSF Communications, and a rigorous cost analysis of the computational complexity associated with each technique was then carried out.

The scenario configuration encountered by a base station depends strongly on the mobile communications system in which it operates. The suitability of different array processing algorithms might vary substantially depending on whether it is operating in a TDMA, FDMA, or CDMA system. In this respect, the main focus of the SUNBEAM project was to characterize the third-generation standards proposed at that time by the European Telecommunications Standards Institute (ETSI) and now part of the IMT-2000 family (i.e., the FDD and TDD modes of UTRA). A common simulation 


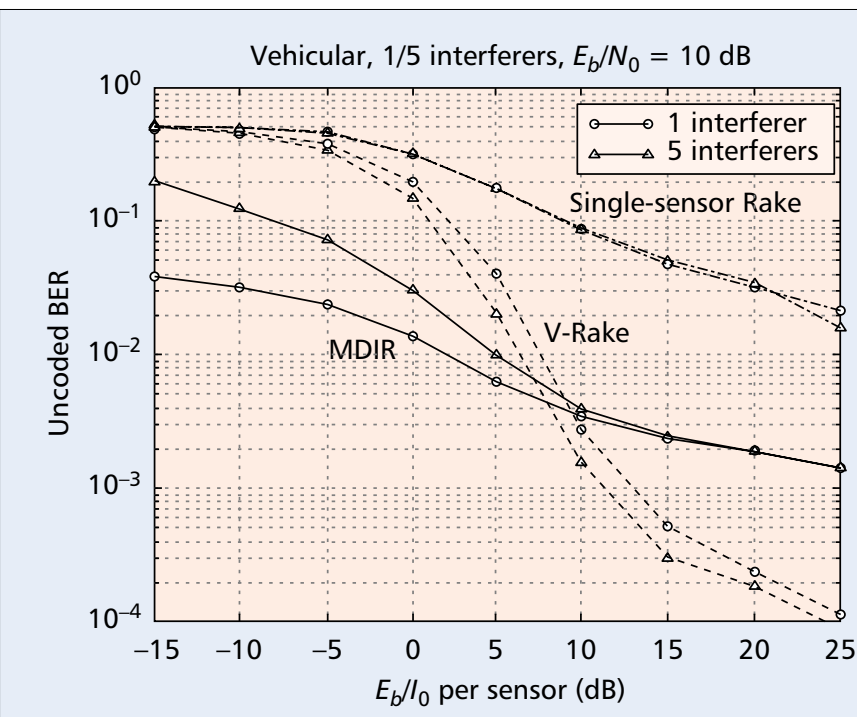

(a)

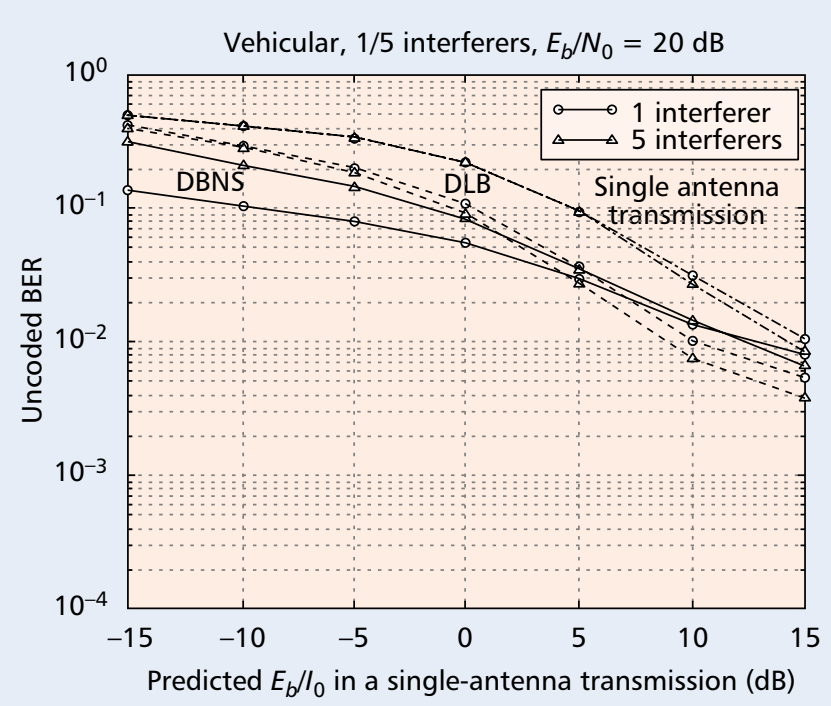

(b)

Figure 5. Link-level performance results for a) uplink and b) downlink of the FDD mode of UTRA (eight antennas).

platform for the evaluation of array processing algorithms at both the link and system levels was developed. Link-level simulations take into account all aspects of the transmission (e.g., modulation format, channel estimation, power control, and interference modeling), but do not incorporate cellular configurations or mobility models. These aspects are usually simulated at a higher level, within the so-called system-level simulators, which basically make use of the results obtained at the link level and do not simply model the physical transmission link. Both types of simulation were performed within the project.

Two different types of array processing algorithm were considered appropriate for the uplink of the FDD mode of UTRA [1]: interfering cancellation schemes (based on spatial filtering or beamforming) and diversity combining schemes (based on rake-receiver technology). Multi-user detectors were discarded right from the beginning because of the asynchronous multirate structure of the uplink signal and the potentially high number of users to be served in a cell. Figure 4 shows a schematic representing the two algorithms simulated in the uplink. The first algorithm, referred to as Vector Rake or VRake, combines the space-time diversity from the multipath environment, disregarding the presence of interference. The second, referred to as MDIR [2], performs a spatial cancellation followed by a Rake receiver. In the downlink, two different beamforming approaches were selected: a simple phased array, where the beam pattern is designed to point in the direction of the user of interest, and a downlink beamforming with null steering which also steered nulls toward the potentially interfered users. In both cases, the downlink weight vector was calculated using uplink measurements, assuming that uplink and downlink frequencies are sufficiently close to disregard the discrepancies between spatial responses.

Figure 5 shows some link-level simulation results for the uplink and downlink in the FDD mode of UTRA operating over a vehicular channel model with a mobile speed of $120 \mathrm{~km} / \mathrm{h}$. In the uplink, interference nulling schemes (MDIR) are appropriate at low values of the $E_{b} / I_{0}$ (high level of directional interference), whereas diversity-combining architectures (V-Rake) seem more appropriate when omnidirectional Gaussian noise prevails. In the downlink, gains obtained as a result of beamforming with null steering (DBNS) toward the potentially interfered users are quite marginal, especially at high mobile speed. The reasons for this behavior are the lack of correlation in uplink and downlink channels in an FDD system and the fact that smaller variations of the channel impulse responses due to the Doppler effect originate a great loss of the beamformer nulling capability. In any case, it seems clear that the increased computational complexity of the null steering technique does not really pay off when compared to a much simpler beam steering (DLB) architecture.

As for the TDD mode of UTRA [3], two different strategies were contemplated in the uplink: multi-user detection techniques, based on either a minimum mean squared error or a zero-forcing implementation, and interference cancellation techniques (the MDIR approach in Fig. 4). V-Rake receivers were not considered because in previous investigations during the TSUNAMI (II) project they were shown to perform worse than beamforming techniques. The two approaches followed in the downlink were equivalent to those selected in the FDD mode.

Figure 6 represents some of the link-level performance results for the uplink of the TDD mode of UTRA in an indoor setting. The best performance is always obtained by multi-user techniques (JD-MMSE). However, interference cancellation schemes should not immediately be discarded, since they provide significant gains with respect to the single-sensor receiver; more 


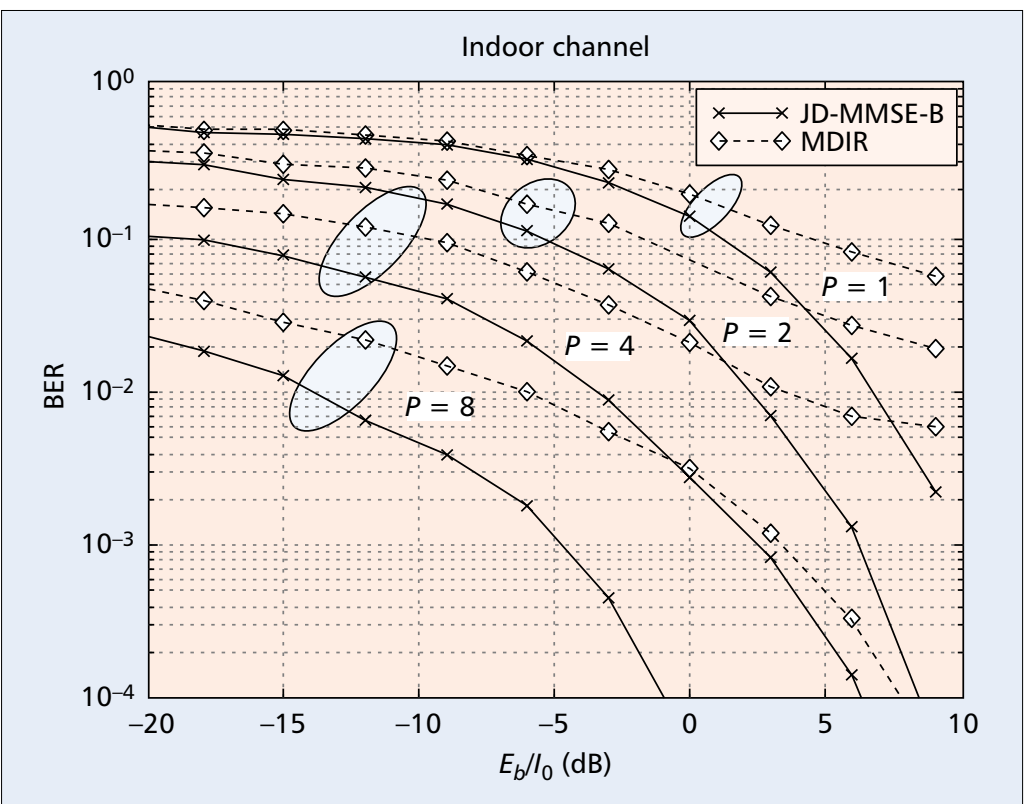

Figure 6. Link-level performance results for the uplink of the TDD mode of UTRA in an indoor environment. $\mathrm{P}$ stands for the number of receiving antennas.

important, the computational load associated with their potential implementation is much lower than the multi-user scheme.

In order to assess the implications of using array processing techniques in terms of coverage and spectral efficiency, system-level simulations were also carried out [4]. For the FDD uplink pedestrian environment (speeds of about 3 $\mathrm{km} / \mathrm{h}$ ), the interference cancellation scheme (MDIR) was shown to give only marginal performance benefits in terms of spectrum efficiency. In the vehicular environment (speeds of about $120 \mathrm{~km} / \mathrm{h}$ ) MDIR was shown to outperform $\mathrm{V}$-Rake in all services, due to the severe nature of the radio channel. In any case, improvements with respect to the single-sensor receiver in terms of both coverage and spectral efficiency were impressive. Namely, cell capacity can be multiplied by factors up to 20 for 144 $\mathrm{kb} / \mathrm{s}$ and above services, which correspond to

\begin{tabular}{|c|c|c|c|c|}
\hline \multirow[t]{2}{*}{ Unit } & \multirow[t]{2}{*}{ Algorithm } & & \multicolumn{2}{|c|}{ Number of sensors } \\
\hline & & 1 & 4 & 8 \\
\hline \multirow{2}{*}{$\begin{array}{l}\text { Millions of C-pseudo- } \\
\text { Macc/s }\end{array}$} & V-Rake & 20 & 70 & 139 \\
\hline & MDIR & $\mathrm{N} / \mathrm{A}$ & 70 & 139 \\
\hline \multirow[t]{2}{*}{ Millions of C-Macc/s } & V-Rake & 12 & 47 & 93 \\
\hline & MDIR & N/A & $27+39 / U^{(i i)}$ & $43+139 / U^{(i i)}$ \\
\hline \multirow{2}{*}{$\begin{array}{l}\text { Millions of Flop-R- } \\
\text { Macc/s }\end{array}$} & V-Rake & 0 & 0 & 0 \\
\hline & MDIR & $\mathrm{N} / \mathrm{A}$ & 4.8 & 21 \\
\hline
\end{tabular}

(i): "C-pseudo-Macc" denotes a complex multiply-accumulate operation with one operand being $\pm 1 \pm i$. Tthis nature of operation appears in channel estimations.

(ii): $U$ denotes the number of users treated in the BTS cell. A typical minimum is 4 , which gives a realistic upper bound.

Table 1. Compared requirements of MDIR and V-Rake. the core value-added services of UMTS. Similar conclusions were also obtained for the TDD mode. As a consequence, preference was given to MDIR.

Since the antenna processing is performed digitally, the proposed techniques offer high scalability vs. the number of antennas and vs. the available baseband processing power. They are also interchangeable with each other or with other reception techniques, such as multi-user detection techniques. This allows dynamic reconfiguration and optimization of hardware resource.

As for complexity, comparative CPU load for the algorithms under consideration (V-Rake and MDIR) have been obtained, which enables realistic design choices about algorithm suitability. Table 1 summarizes the comparison [5].

This table shows that the algorithms exhibit comparable complexities, the extra cost of MDIR synthesis being balanced by its reduced implementation complexity, as depicted in Fig. 4. The values given in the table correspond to a baseband architecture utilizing floating point processors. Nonetheless, algorithmic optimization can also be done to use fixed point arithmetic with the corresponding DSP throughput increase. Note also that the processing requirements expressed in floating point operations are upper bounds corresponding to the matrix algebra manipulations. Keeping in mind that, for instance, the Texas Instrument high-end C6203 DSP has a peak processing capacity of 150 millions C-pseudo-Macc/s (Table 1, note i), it can be seen that servicing one user becomes possible with four antennas and less than one processor. Furthermore, utilizing field programmable gate arrays (FPGAs) for the channel estimation stage can further optimize the hardware design.

The preceding results show that for UMTS FDD and TDD uplink, the MDIR algorithm enables us to reach very high cost efficient capacity increases. Therefore, this algorithm can be considered as a key technique for third-generation base stations. In contrast to most of the smart antennas techniques which use analog technologies (e.g., Buttler matrices), this digital processing-based scheme is fully compatible with the flexibility and scalability the software radio base station requires.

\section{CONCLUSIONS}

Both first- (analog) and second-generation cellular communication systems were designed under the implicit assumption that only conventional antennas were going to be used. It was only recently that these systems have been able to benefit from adaptive antenna technology, but still in a suboptimal manner due to "single-antenna-oriented" system design constraints. The standardization process for thirdgeneration systems, in particular the activities that led to the current UTRA specifications, has been well aware of the potential benefits of adaptive antenna technologies. Adaptive antenna operation is included in UTRA as a capacity enhancement option for which appropriate link adaptation mechanisms are provisioned. 
Still, third-generation specifications are far from being frozen and will be updated, on a yearly basis paving the way toward what is currently known as "systems beyond 3G." Within this continuously changing environment it is apparent that base station design must be flexible in nature and able to accommodate, preferably by soft reconfiguration, at least different modes or specifications of one or several standards. In this context, the SUNBEAM project studied the additional requirements implied by adaptive antennas in software radio base stations in order to ensure that flexible technological constraints would not prevent their potential benefits. As such, the project activities covered a wide range of adaptive antenna related aspects, from rigorous performance analysis of different algorithmic options to architectural and implementation requirements of these techniques.

The work undertaken in SUNBEAM added new consistent elements to support adaptive antenna introduction in $3 \mathrm{G}$ base stations, taking into account multiple standard requirements.

\section{REFERENCES}

[1] C. Anton-Haro, J. R. Fonollosa, and X. Mestre, "Algorithms for Flexible Multi-Standard Array Processing: FDD Mode of UTRA," 4th ACTS Mobile Commun. Summit '99, Sorrento, Italy, June 1999.

[2] M. A. Lagunas, J. Vidal, and A. I. Pérez-Neira, "Joint Array Combining and MLSE for Single-User Receivers in Multipath Gaussian Multiuser Channels," IEEE JSAC, vol. 18, no. 11, Nov. 2000.

[3] C. Anton-Haro, J. R. Fonollosa, and X. Mestre, "Algorithms for Flexible Multi-Standard Array Processing: TDD Mode of UTRA," 4th ACTS Mobile Commun. Summit '99, Sorrento, Italy, June 1999.
[4] D. Brooks, "System Level Performance Evaluation of Flexible Array Processing Algorithms," 4th ACTS Mobile Commun. Summit '99, Sorrento, Italy, June 1999.

[5] E. Nicollet and J. Thibault, "Cost Efficient Baseband Smart Antennas for UMTS Uplink: MDIR Algorithm," IST Mobile Commun. Summit 2000, Galway, Ireland, Oct. 2000.

\section{BIOGRAPHIES}

ANA I. PÉREZ-NeIRA [S'92-M'95] (anuska@gps.tsc.upc.es) received her Ph.D. in electronics engineering from the Universitat Politècnica de Catalunya (UPC), Barcelona, Spain, in 1995. In 1991 she joined the Department of Signal Theory and Communication of the UPC. In 1992 she became lecturer, and since 1996 she has been an associate professor at UPC, where she teaches and coordinates graduate and undergraduate courses. Her current research interests are in statistical array processing and fuzzy processing, with applications to mobile/satellite communication systems. She has participated in national research projects and in European ACTS and IST projects.

XAVIER MESTRE [S'96] (mestre@gps.tsc.upc.es) received his electrical engineering degree from UPC, Spain, in 1997. After graduating, he joined the Communications Signal Processing Group at the same university, where he has been working as a research assistant. He has participated actively in the EC-funded projects ACTS TSUNAMI-II, ACTS SUNBEAM, and IST METRA, and is planning to obtain his Ph.D. by the end of 2001.

JAVIER ROdRIguez Fonollosa [S'90-M'92-SM'98] (fono@gps.tsc.ups.es) received his Ph.D. in electrical and computer engineering at Northeastern University, Boston, Masachusetts, in 1992. In 1993 he joined UPC, Barcelona, Spain, where he is now associate professor. He is the author of nine journal and about 50 conference papers in the areas of signal processing and communications. Since 1995 he has led UPC's participation in the European Commission funded ACTS Mobile projects TSUNAMI(II) and SUNBEAM which include the analysis of adaptive antennas in $2 \mathrm{G}$ and $3 \mathrm{G}$ cellular mobile communication systems. He is currently project coordinator of the IST METRA project. He is a member of the Signal Processing for Communications (SPCOM) Technical Committee of the IEEE Signal Processing Society.
Third generation

specifications are

far from being

frozen, and will

be updated on a

yearly basis

paving the way

toward what is

currently known

as "systems

beyond 3G." 